\title{
The emergence of classical behavior in magnetic adatoms
}

\author{
F. Delgado, ${ }^{1}$ S.Loth, ${ }^{2,3}$ M. Zielinski, ${ }^{4}$ and J. Fernández-Rossier ${ }^{1}$ \\ ${ }^{1}$ International Iberian Nanotechnology Laboratory (INL), \\ Av. Mestre José Veiga, 4715-310 Braga, Portugal \\ ${ }^{2}$ Max Planck Institute for the Structure and Dynamics of Matter, Hamburg, Germany \\ ${ }^{3}$ Max Planck Institute for Solid State Research, Stuttgart, Germany \\ ${ }^{4}$ Institute of Physics, Faculty of Physics, Astronomy and Informatics, \\ Nicolaus Copernicus University, Grudziadzka 5, 87-100 Torun, Poland
}

(Dated: May 15, 2014)

\begin{abstract}
A wide class of nanomagnets shows striking quantum behavior, known as quantum spin tunneling (QST): instead of two degenerate ground states with opposite magnetizations, a bondingantibonding pair forms, resulting in a splitting of the ground state doublet with wave functions linear combination of two classically opposite magnetic states, leading to the quenching of their magnetic moment. Here we study how QST is destroyed and classical behavior emerges in the case of magnetic adatoms, as the strength of their coupling, either to a bath or to each other, is increased. Both spin-bath and spin-spin coupling renormalize the QST splitting to zero allowing the environmental decoherence to eliminate superpositions between classical states, leading to the emergence of spontaneous magnetization.
\end{abstract}

Understanding how matter, governed by quantum mechanics at the atomic scale, behaves with classical rules at the macroscale is one of the fundamental open questions in physics [1 3]. One of the most drastic manifestations of the quantum character is found when a system is prepared in a linear combination of two classically different states. In magnetic systems, such a quantum state results in the phenomenon of quantum spin tunneling (QST) [4, inducing an energy splitting $\Delta_{0}$ between the two states with opposite magnetization and quenching its average magnetization.

Attending to the nature of their ground state, nanoscale quantized spin systems can be classified in two groups, see Figs. 1a,b. Type $\mathrm{C}$ systems, such as single half-integer spins or antiferromagnetic chains of Ising coupled spins, have two degenerate ground states with wave functions $\left|C_{1}\right\rangle$ and $\left|C_{2}\right\rangle$ that correspond to states with opposite magnetizations. Type Q systems, such as anisotropic single integer spins, have a unique ground state $\left|\phi_{G}\right\rangle$, as well as a first excited state $\left|\phi_{X}\right\rangle$, both satisfying

$$
|\phi\rangle=\left|C_{1}\right\rangle+e^{i \theta}\left|C_{2}\right\rangle
$$

where $\theta$ is a phase. Whereas both correspond to quantum spins, only type $\mathrm{Q}$ systems depart radically from the classical picture of a nanomagnet because the quantum expectation value $\langle\phi|\vec{S}| \phi\rangle$ of the atomic spin operator $\vec{S}$ vanishes identically, and not only in the statistical sense. The two degenerate ground states of type $\mathrm{C}$ systems could be prepared in superposition states like (1), but coupling to the environment would rapidly lead to decoherence, restoring the classical behavior with two ground states with opposite magnetization [5]. In contrast, in type $\mathrm{Q}$ systems the coherent superposition is built-in dynamically, and it is protected by the energy separation $\Delta_{0}$ between $\left|\phi_{G}\right\rangle$ and $\left|\phi_{X}\right\rangle$, see Fig. 1b. Ex- amples of type $\mathrm{Q}$ magnets are found in transition metal impurities in insulators [6], spin color centers [7, magnetic molecules [8] and single molecule magnets [4].

Here we focus on magnetic atoms deposited on conducting surfaces 9 12, where scanning tunneling microscopes (STM) can probe the two quantities that characterize quantum or classical behavior: the quantum spin tunneling splitting $\Delta$, which can be measured by inelastic electron tunneling spectroscopy [8, 9, 11, and their magnetization, accessible through spin polarized STM [13.

It has been found that diverse magnetic adatoms can be described with the spin Hamiltonian: 4, 9, 11, 14

$$
\mathcal{H}_{\mathrm{S}}=D \hat{S}_{z}^{2}+E\left(\hat{S}_{x}^{2}-\hat{S}_{y}^{2}\right) .
$$

This Hamiltonian yields a type Q spectrum for integer spins $S$ with negative uniaxial anisotropy $D<0$ and finite in-plane anisotropy $E$. In that case, both the nondegenerate ground state $\left|\phi_{G}\right\rangle$ and the first excited state $\left|\phi_{X}\right\rangle$, split by $\Delta_{0} \propto E(E / D)^{S-1}$, satisfy Eq. (1) with $\left|C_{1}\right\rangle \approx|+S\rangle$ and $\left|C_{2}\right\rangle \approx|-S\rangle$ (see Fig. 1a). This Hamiltonian correctly accounts for the observed $d I / d V$ spectra of Fe adatoms on $\mathrm{Cu}_{2} \mathrm{~N} / \mathrm{Cu}(100)$ 9], Fe Phthalocyanine $(\mathrm{FePc})$ molecules on $\mathrm{CuO} / \mathrm{Cu}(110)[8]$ and $\mathrm{Fe}$ adatoms on InSb [11] (with $S=2$ for $\mathrm{Fe} / \mathrm{Cu}_{2} \mathrm{~N}$ and $S=1$ for the others). In these three systems the $d I / d V$ spectra reveal finite quantum spin tunneling between $\left|\phi_{G}\right\rangle$ and $\left|\phi_{X}\right\rangle$ and a null magnetic moment can be expected.

Spin polarized STM magnetometry on short chains of $\mathrm{Fe}$ atoms on $\mathrm{Cu}_{2} \mathrm{~N} / \mathrm{Cu}(100)$ are not able 15 to detect magnetic moment, consistent with a type $\mathrm{Q}$ behavior and the observation of QST splitting on the single atom. Intriguingly, longer chains display a spontaneous atomic magnetization, in the form of antiferromagnetically aligned Néel states 15 .

This paper is devoted to understanding how the conventional classical picture of a magnet with two equiva- 

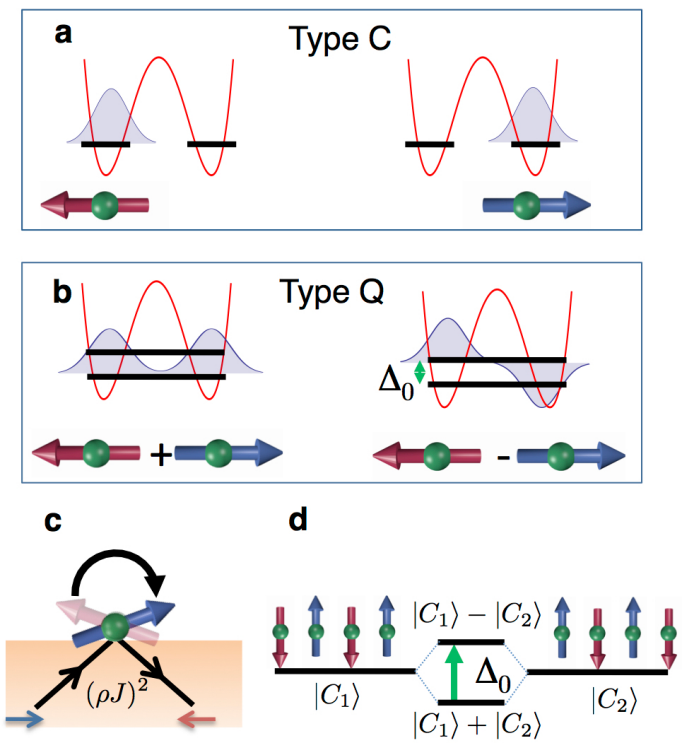

FIG. 1. Two types of quantized spin systems. (a) Scheme of a type $\mathrm{C}$ spin system, with an easy axis and two degenerate ground states, bearing each a finite magnetic moment. (b) Scheme of a type Q spin system. Due to QST, bonding and antibonding linear combination of states with opposite magnetization, are formed and are separated in energy by $\Delta_{0}$, the QST splitting. (c) Scheme of Kondo exchange interaction between a single magnetic atom and conduction electrons that quenches $\Delta_{0}$ (see Fig. 2) as $(\rho J)$, the product of the density of states of conducting electrons and the Kondo exchange, is increased. (d) Representation of the two classical degenerate Néel states for spin chains, denoted as $\left|C_{1}\right\rangle$ and $\left|C_{2}\right\rangle$, as well as the type Q bonding and anti-bonding states and their corresponding QST splitting.

lent ground states with opposite magnetization emerges for type Q magnetic adatoms. We discuss two independent mechanisms: Kondo exchange, that operates even for a single magnetic adatom, and interatomic exchange. The driving factor in both cases is the quenching of the QST splitting so that the dressed type Q system becomes effectively a type $\mathrm{C}$ exhibiting two classical degenerate ground states.

We consider first Kondo exchange in the the weak coupling regime, where the magnetic adatom spin preserves its identity and the Kondo singlet has not been formed. In that limit, perturbation theory [16] predicts that Kondo exchange produces both a broadening $\Gamma$ and a shift of the atomic spin excitations, in agreement with experiments [17, 18]. Both quantities are proportional to the dimensionless constant $(\rho J)^{2}$, the product of the density of states $\rho$ of the surface electrons and the Kondo exchange $J$. Here we go beyond perturbative theory and show that a sufficiently large $\rho J$ quenches completely the QST. To do so, we assume that the separation of the ground stated doublet from the higher excited states is larger than all relevant energy scales, such as thermal en- ergy or the QST, so that we truncate the Hilbert space keeping only the two lowest energy states, $\left|\phi_{G}\right\rangle$ and $\left|\phi_{X}\right\rangle$. Hence, defining a pseudo-spin $1 / 2$, with Pauli matrices $\vec{\tau}$, the atomic spin operator represented in this space takes the form:

$$
\left(\hat{S}_{x}, \hat{S}_{y}, \hat{S}_{z}\right) \rightarrow\left\langle\phi_{G}\left|\hat{S}_{z}\right| \phi_{X}\right\rangle\left(0,0, \hat{\tau}_{x}\right) .
$$

This means that the exchange coupling acts only through the $S_{z}$-conserving (Ising) channel, which prevents the formation of a Kondo singlet, but creates a pseudo-spin flip in the space of $\left|\phi_{G}\right\rangle$ and $\left|\phi_{X}\right\rangle$. As a consequence, the Kondo Hamiltonian projected in the $\left(\phi_{G}, \phi_{X}\right)$ subspace has the form

$$
H_{\mathrm{K}} \equiv \sum_{\vec{k}, \sigma} \epsilon_{|\vec{k}|} c_{\vec{k}, \sigma}^{\dagger} c_{\vec{k} \sigma}+\frac{\Delta_{0}}{2} \hat{\tau}_{z}+\hat{\tau}_{x} \sum_{\vec{k}, \vec{k}^{\prime}} \frac{j}{2}\left(c_{\vec{k}, \uparrow}^{\dagger} c_{\overrightarrow{k^{\prime}} \uparrow}-c_{\vec{k}, \downarrow}^{\dagger} c_{\vec{k}^{\prime} \downarrow}\right),
$$

where $j=J\left\langle\phi_{G}\left|\hat{S}_{z}\right| \phi_{X}\right\rangle$. This Hamiltonian is known as the Ising-Kondo model in a transverse field [19].

For a point scatterer, conduction electrons can be described as one dimensional fermions, which permits making use of the bosonization technique [20] where charge and spin densities are represented in terms of bosonic operators $b_{k}, b_{k}^{\dagger}[21,22$. This allows mapping the original Kondo model for the type Q spins into the spin-boson (SB) Hamiltonian with an Ohmic spectral density [22, 23.

$$
\begin{aligned}
H_{S B}= & \frac{\Delta_{0}}{2} \hat{\tau}_{z}+\hbar v_{F} \sum_{k>0} k b_{k}^{\dagger} b_{k} \\
& +\hbar v_{F} \hat{\tau}_{x} \sqrt{\pi \alpha} \sum_{k} \sqrt{\frac{|k|}{L}} e^{-k v_{F} / 2 \omega_{c}}\left(b_{k}^{\dagger}+b_{k}\right),
\end{aligned}
$$

where $v_{F}$ is the Fermi velocity, $L$ is the size of the system, and $\hbar \omega_{c}$ is the bosonic energy cut-off. Here the first term describes the QST of the bare magnetic atom, the second the surface electrons, and the third term accounts for the Kondo interaction. The constant $\alpha=(\rho J)^{2}\left|\left\langle\phi_{G}\left|S_{z}\right| \phi_{X}\right\rangle\right|^{2}$ plays a key role in the SB model (see Supplemental information for details).

The SB Hamiltonian is a paradigm model to describe a quantum to classical phase transition induced by environmental decoherence [22]. The transition is driven by the competition between QST (with strength $\Delta_{0}$ ), which favors the mixing of states with opposite $S_{z}$, and the Kondo coupling (with strength $\alpha$ ) that favors localization of the system in one of the two states with $S_{z}= \pm S$. The SB model yields the following non-perturbative result for the renormalization of the QST splitting $\Delta$ at zero temperature [22, 24]:

$$
\frac{\Delta}{\Delta_{0}} \approx(1-\Theta(\alpha))\left(\frac{\Delta_{0}}{\hbar \omega_{c}}\right)^{\frac{\alpha}{1-\alpha}}
$$

where $\Theta$ is the step function. Hence, increasing $\alpha$ decreases $\Delta / \Delta_{0}$ exponentially fast, as shown in Fig. 2, 


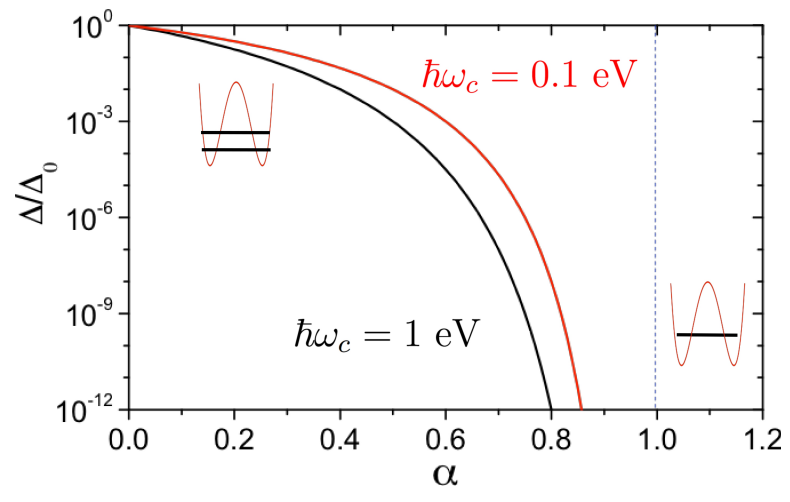

FIG. 2. Renormalization of the QST splitting for a Kondo coupled spin. Zero-temperature renormalized splitting $\Delta$ in units of the unperturbed level splitting $\Delta_{0}$, Eq. (5), of a type Q single spin Kondo-coupled to an electron gas as function of coupling strength. Red (Black) lines show two different energy cut-offs, $\hbar \omega_{c}\left(\Delta_{0}=1 \mathrm{meV}\right) . \Delta$ is very rapidly quenched (notice logarithmic scale) and vanishes identically for $\alpha \rightarrow 1$.

vanishing completely when $\alpha \geq 1$. This point marks a zero-temperature quantum phase transition beyond which quantum tunneling is suppressed.

Importantly, IETS measurements permits inferring $\alpha$. The full width at half maximum $W_{2,1}$ of the peak (or dip) in the experimental $d^{2} I / d V^{2}$, corresponding to the $\left|\phi_{1}\right\rangle \rightarrow\left|\phi_{2}\right\rangle$ transition, is related to the intrinsic relaxation rate $\Gamma_{2,1} / \hbar$ via 25 .

$$
\Gamma_{2,1}=\sqrt{e^{2} W_{2,1}^{2}-\left(1.7 e V_{A C}\right)^{2}-\left(5.4 k_{B} T\right)^{2}},
$$

where $V_{A C}$ is the modulation voltage in the applied bias. In addition, and within perturbation theory, the exchange-induced energy broadening $\Gamma_{2,1}$ is given by [16]

$$
\Gamma_{2,1}=\alpha \frac{\pi}{2} \Delta_{21}\left[1+n_{B}\left(\Delta_{21}\right)\right] \frac{\sum_{a}\left|\left\langle\phi_{1}\left|\hat{S}_{a}\right| \phi_{2}\right\rangle\right|^{2}}{\left|\left\langle\phi_{G}\left|S_{z}\right| \phi_{X}\right\rangle\right|^{2}},
$$

where $\Delta_{21}$ is the energy of the transition and $n_{B}$ is the thermal Bose factor. Thus, from the experimental results we find that, while $\mathrm{Fe}$ on $\mathrm{Cu}_{2} \mathrm{~N} / \mathrm{Cu}(100)$ has $\alpha<0.1$ and a finite $\Delta, \mathrm{Fe}$ on $\mathrm{Cu}(111)$ has $\alpha \approx 2$, leading to $\Delta=0$ even if symmetry-breaking effects or higher-order anisotropy contributions remove the degeneracy caused by the $C_{3 v}$ symmetry [12, 26] of the surface.

In the SB model at finite temperature, spin Rabi oscillations, and therefore the existence of non-degenerate superposition ground states, are suppressed when $\Delta /\left(k_{B} T\right) \lesssim 2 \alpha 22$. This enables to place the experimental observations in a phase diagram $\left(\alpha, \Delta / k_{B} T\right)$, shown in Fig. 3, separating quantum and classical regions.

We now address the emergence of classical behaviour in chains of spin-coupled atoms. Sufficiently long chains of Fe atoms present classical behaviour (type C) with two

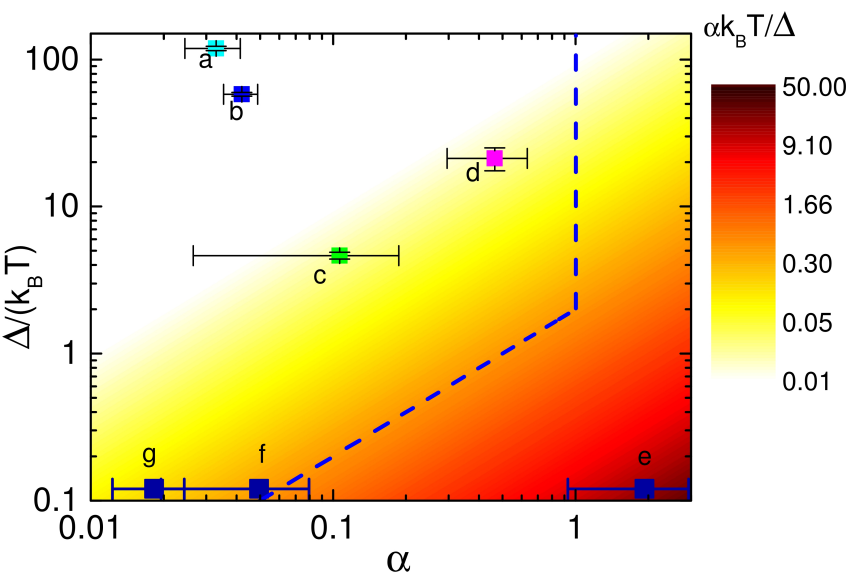

FIG. 3. Finite-temperature phase diagram. The phase space spanned by the renormalized QST splitting $\Delta$ in units of $k_{B} T$ and the substrate coupling constant, $\alpha$, is classified into Quantum (where $\alpha k_{B} T / \Delta \ll 1$ ), and Classical $\left(\alpha k_{B} T / \Delta \gg 1\right.$ ) regimes. The blue dashed line indicates the limiting condition $\alpha=\operatorname{Min}\left[\Delta /\left(2 k_{B} T\right), 1\right]$. Experimentally reported type $\mathrm{Q}$ spin systems: (a),(b) Fe-Phthalocyanine molecules on $\mathrm{CuO} / \mathrm{Cu}(110)$ 8, (c) Fe atoms on $\mathrm{Cu}_{2} \mathrm{~N} / \mathrm{Cu}(100)$ 9], and (d) Fe dopants on $\operatorname{InSb}(110)$ [10. Measured type C systems, where $\Delta$ could not be determined experimentally, are shown over the horizontal axis: (e) $\mathrm{Fe}$ atoms on $\mathrm{Cu}(111)$ [11, and (g) and (f) Fe atoms on Pt(111) [12.

degenerate Néel states, whereas isolated Fe atoms show QST [15. Here Fe-Fe exchange causes a renormalization of the QST splitting even in the absence of Kondocoupling to the substrate conduction electrons. We use the following Hamiltonian for $N$ spins:

$$
\mathcal{H}=\sum_{n=1}^{N} \mathcal{H}_{\mathrm{S}}(n)+J_{\mathrm{H}} \sum_{n=1}^{N-1} \vec{S}(n) \cdot \vec{S}(n+1),
$$

where the first term describes the single ion Hamiltonian of Eq. (2) for each Fe, and the second their antiferromagnetic exchange $\left(J_{H}>0\right)$. When acting independently, both terms yield a unique ground state without spontaneous magnetization. However, their combination gives non-trivial results. This can be first seen using the same truncation scheme of the single atom case, keeping only 2 levels per site. Hamiltonian (7) then maps into the quantum Ising model with a transverse field (QIMTF) (more details in Supplementary information):

$$
\mathcal{H} \equiv \sum_{n=1}^{N} \frac{\Delta_{0}}{2} \hat{\tau}_{z}(n)+j_{H} \sum_{n=1}^{N-1} \hat{\tau}_{x}(n) \hat{\tau}_{x}(n+1)
$$

where $j_{H}=J_{H}\left|\left\langle\phi_{G}\left|\hat{S}_{z}\right| \phi_{X}\right\rangle\right|^{2}$. This model can be solved exactly and presents a quantum phase transition in the thermodynamic limit $(N \rightarrow \infty)$, separating a type $\mathrm{C}$ from a type $\mathrm{Q}$ phase. In terms of the dimensionless parameter $g \equiv 2 j_{H} / \Delta_{0}$, the transition occurs at $g_{c}=1[27$. 
For $g<1$ the spin chain is in a quantum paramagnetic phase with a unique ground state and $\left\langle\hat{\tau}_{x}(n)\right\rangle=$ $\left\langle\hat{S}_{z}(n)\right\rangle=0$. For $g \geq 1$, it is in a magnetically ordered phase, with 2 equivalent ground states with staggered magnetization, $\left\langle\hat{\tau}_{x}(n)\right\rangle \propto\left\langle\hat{S}_{z}(n)\right\rangle \propto(-1)^{n}$. In this thermodynamic limit, the QST is renormalized by the interactions according to 27.

$$
\frac{\Delta}{\Delta_{0}}=|1-g| \Theta(1-g) .
$$

This result shows that, as in the case of Kondo exchange in Eq. (5), interatomic exchange also renormalizes QST, and when sufficiently strong, suppresses it completely. However, direct application of Eqs. (8) and (9) is not possible for systems where exchange and anisotropy are of the same order, $J_{H} \sim|D|$, preventing the use of the mapping to an Ising model. Instead, we compute the eigenstates of Hamiltonian (7) numerically, and compare with those of finite size chains of the QIMTF (Fig. 4). In both systems we find the same phenomenology: both the ground and first excited states, $\left|\phi_{G}\right\rangle$ and $\left|\phi_{X}\right\rangle$, satisfy Eq. (1) with $\left|C_{1}\right\rangle$ and $\left|C_{2}\right\rangle$ being classical Néel-like states, and the next excited states lie much higher in energy. Importantly, it is still true that the QST splitting is renormalized by the interatomic exchange (inset of Fig. 4a). Crucially, for the observed value $J_{H}=0.7 \mathrm{meV}$ [15, 28], the quenching of $\Delta$ increases exponentially with the size of the chain (Fig. 4b).

In rigor, interatomic exchange in finite chains renormalizes the QST to a tiny but finite value (see Fig.4). Therefore, the observed [15] emergence of classical behavior is probably assisted as well by the Kondo coupling. Using results from second order perturbation theory, one finds that the Kondo-induced decoherence rate of a chain of $N$ spins is

$$
T_{2}^{-1}(N)=\frac{N \pi}{2} \alpha S^{2} \frac{k_{B} T}{\hbar} .
$$

For instance, the $N=8 \mathrm{Fe}$ chain of Ref. [15] leads to $\Delta /\left(\hbar T_{2}^{-1}\right) \lesssim 10^{-6}$ at $T=0.5 \mathrm{~K}$, indicating that the $\mathrm{Fe}$ chain will be in the decohered type $\mathrm{C}$ state. Thus, the combination of interatomic exchange, that reduces almost down to zero the QST of the monomer, and the enhanced spin decoherence of the chain due to Kondo exchange with the substrate, lead to the emergence of classical behaviour of the finite size spin chains.

Our results provide a general scenario for the emergence of classical magnetism in quantum spins systems that have a unique ground state superposition of classical states with opposite magnetizations. A sufficiently strong coupling to either the itinerant electrons or to other localized spins, leads to a phase with a doubly degenerate ground state where classical behaviour appears. Using experimentally verified coupling strengths we find that this transition can occur for small ensembles of interacting atoms $(N<10)$ or even individual atoms. Hence, a
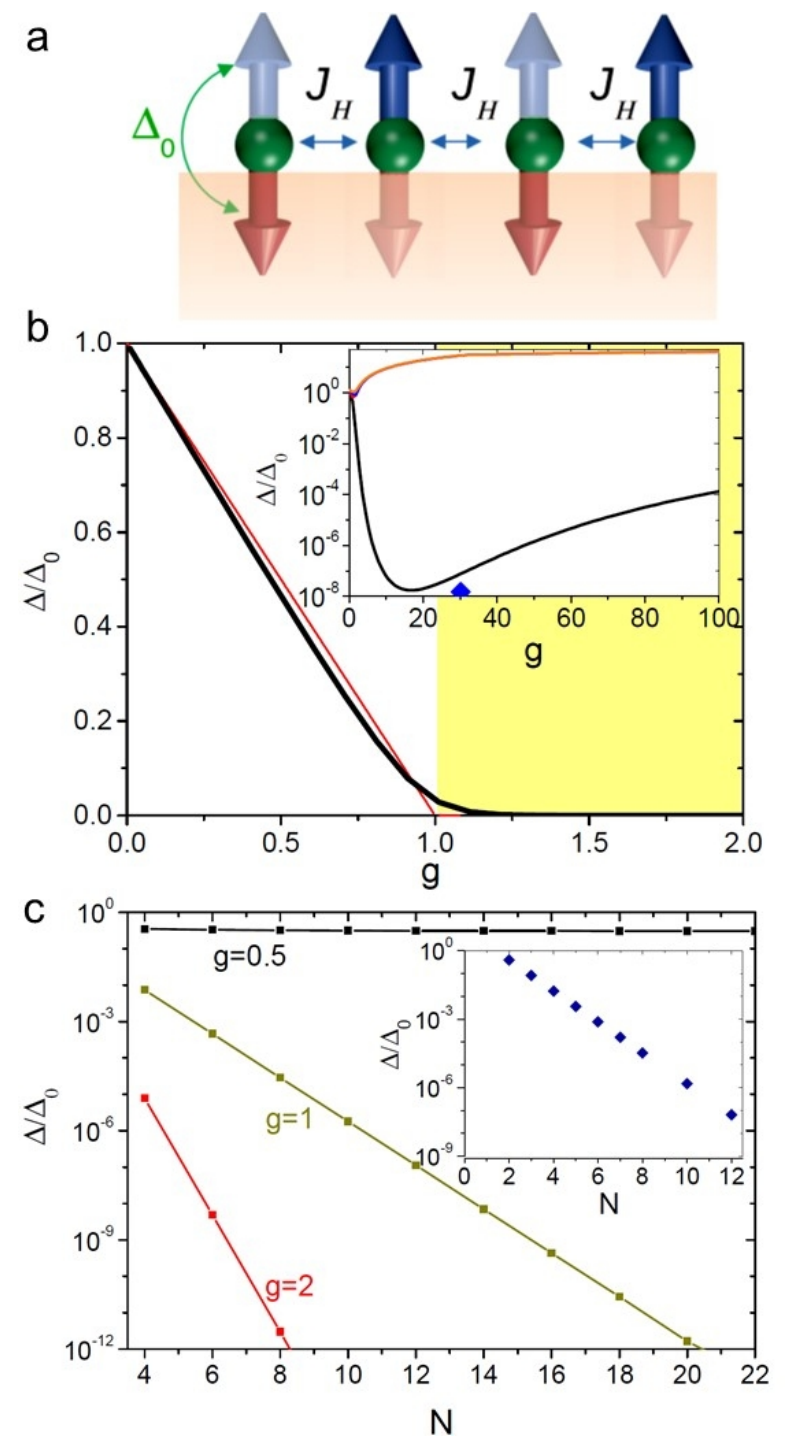

FIG. 4. Quenching of quantum spin tunneling in spin chains. (a) Schematic of a superposition state of two Néel states in a finite antiferromagnetic spin chain. (b) QST splitting of a $S=1 / 2$ Ising chain in a transverse field, Eq. (8), versus the dimensionless exchange coupling $g=2 j_{H} / \Delta_{0}$ for a finite $N=20$ chain (black line) and the infinite chain (red). The infinite chain has a quantum phase transition to the classical (shaded) region at $g=1$. The inset shows the QST splitting of the $S=2$ Heisenberg spin chain together with higher energy excitations (orange lines) versus exchange coupling for $\mathrm{Fe}$ chains with $D=-1.5 \mathrm{meV}$ and $E=0.3 \mathrm{meV}$ [15, 28] (the diamond marks the experimental condition where $g \approx 27$ ). (c) Chain size dependence of $\Delta$ in the QIMTF for Ising spin coupling, for $g=0.5<g_{c}$ (weak size dependence), and $g=1,2$ (exponential dependence that leads to a type Q ground state for large $N$ ). Inset: size dependence of $\Delta$ for Hamiltonian (9) with the experimental parameters [15, 28, showing an exponential dependence analogous to the Ising case with $g>1$ (type C system). 
the classical phase in nanomagnets appears as a quantum phase transition to a quantum decohered phase.

Acknowledgements We acknowledge A. J. Heinrich, R. Aguado, M. A. Cazalilla and A. Khajetoorians for fruitful discussions. This work has been financially supported by Generalitat Valenciana, grant Prometeo 201211.

[1] P. W. Anderson, Science 177, 393 (1972).

[2] W. H. Zurek, Physics Today 44, 36 (1991).

[3] W. H. Zurek, Rev. Mod. Phys. 75, 715 (2003).

[4] D. Gatteschi, R. Sessoli, and J. Villain, Molecular nanomagnets (Oxford University Press, New York, 2006).

[5] F. Delgado and J. Fernández-Rossier, Phys. Rev. Lett. 108, 196602 (2012).

[6] A. Abragam and B. Bleaney, Electron Paramagnetic Resonance of Transition Ions (Oxford University Press, Oxford, 1970).

[7] L. Childress, M. V. Gurudev Dutt, J. M. Taylor, A. S. Zibrov, F. Jelezko, J. Wrachtrup, P. R. Hemmer, and M. D. Lukin, Science 314, 281 (2006).

[8] N. Tsukahara, K. Noto, M. Ohara, S. Shiraki, N. Takagi, Y. Takata, J. Miyawaki, M. Taguchi, A. Chainani, S. Shin, et al., Phys. Rev. Lett. 102, 167203 (2009).

[9] C. Hirjibehedin, C.-Y. Lin, A. Otte, M. Ternes, C. P. Lutz, B. A. Jones, and A. J. Heinrich, Science 317, 1199 (2007).

[10] A. A. Khajetoorians, S. Lounis, B. Chilian, A. T. Costa, L. Zhou, D. L. Mills, J. Wiebe, and R. Wiesendanger, Phys. Rev. Lett. 106, 037205 (2011).

[11] A. A. Khajetoorians, B. Chilian, J. Wiebe, S. Schuwalow, F. Lechermann, and R. Wiesendanger, Nature 467, 1084
$(2010)$

[12] A. A. Khajetoorians, T. Schlenk, B. Schweflinghaus, M. dos Santos Dias, M. Steinbrecher, M. Bouhassoune, S. Lounis, J. Wiebe, and R. Wiesendanger, Phys. Rev. Lett. 111, 157204 (2013).

[13] R. Wiesendanger, Rev. Mod. Phys. 81, 1495 (2009).

[14] A. Gruber, A. Dräbenstedt, C. Tietz, L. Fleury, J. Wrachtrup, and C. Von Borczyskowski, Science 276, 2012 (1997).

[15] S. Loth, S. Baumann, C. P. Lutz, D. M. Eigler, and A. J. Heinrich, Science 335, 196 (2012).

[16] F. Delgado, J. J. Palacios, and J. Fernández-Rossier, Phys. Rev. Lett. 104, 026601 (2010).

[17] J. C. Oberg, M. R. Calvo, F. Delgado, M. Moro-Lagares, D. Serrate, D. Jacob, J. Fernández-Rossier, and C. F. Hirjibehedin, Nature Nanotechnology 9, 64 (2014).

[18] S. Loth, K. von Bergmann, M. Ternes, A. F. Otte, C. P. Lutz, and A. J. Heinrich, Nature Physics 6, 340 (2010).

[19] A. E. Sikkema, W. J. L. Buyers, I. Affleck, and J. Gan, Phys. Rev. B 54, 9322 (1996).

[20] K. D. Schotte and U. Schotte, Phys. Rev. 182, 479 (1969).

[21] D. Mattis and E. Lieb, J. Math. Phys. 6, 304 (1965).

[22] A. J. Leggett, S. Chakravarty, A. T. Dorsey, M. P. A. Fisher, A. Garg, and W. Zwerger, Rev. Mod. Phys. 59, 1 (1987).

[23] A. J. Bray and M. A. Moore, Phys. Rev. Lett. 49, 1545 (1982).

[24] K. Hur, Annals of Physics 323, 2208 (2008).

[25] L. Lauhon and W. Ho, Review of Scientific Instruments 72, 216 (2001).

[26] T. Miyamachi, T. Schuh, T. Märkl, C. Bresch, T. Balashov, A. Stöhr, C. Karlewski, S. André, M. Marthaler, M. Hoffmann, et al., Nature 503, 242 (2013).

[27] P. Pfeuty, Annals of Physics 57, 79 (1970).

[28] B. Bryant, A. Spinelli, J. J. T. Wagenaar, M. Gerrits, and A. F. Otte, Phys. Rev. Lett. 111, 127203 (2013). 